\title{
Letters
}

\section{A Fast High-Order Solver for EM Scattering from Complex Penetrable Bodies: TE Case}

Oscar P. Bruno and Alain Sei

\begin{abstract}
We present a new high-order integral algorithm for the solution of scattering problems by heterogeneous bodies under TE radiation. Here, a scatterer is represented by a (continuously or discontinuously) varying refractive index $n(x)$ within a two-dimensional (2-D) bounded region; solutions of the associated Helmholtz equation under given incident fields are then obtained by high-order inversion of the Lippmann-Schwinger integral equation. The algorithm runs in $\mathcal{O}(N \log (N))$ operations, where $N$ is the number of discretization points. Our method provides highly accurate solutions in short computing times, even for problems in which the scattering bodies contain complex geometric singularities.
\end{abstract}

Index Terms-Electromagnetic (EM) scattering, iterative methods, nonhomogenous media.

\section{INTRODUCTION}

We present a new high-order integral algorithm for the solution of problems of electromagnetic (EM) scattering requiring two-dimensional (2-D) integration. We restrict ourselves to the simplest EMs problem in which 2-D singular integrals occur: scattering by a 2-D heterogeneous body under TE polarized radiation. A basic element in our method is the use of truncated Fourier expansions of the Green function of the problem. The logarithmic point-singularities of the Green function manifest themselves, in the finite Fourier approximations, as singular arcs inside the integration domain; see (3). Such singularity distributions allow for computation of the required 2-D integrals by means of simple 1-D high-order integrators. The algorithm is fast: it runs in $\mathcal{O}(N \log (N))$ operations, where $N$ is the number of discretization points. It can be applied to general configurations, including scatterers in which the refractive index varies discontinuously. An example is provided of a highly singular scattering configuration containing cusps, for which solutions with errors of order $10^{(-5)}$ are obtained in a 13-s computation on a desktop computer. Thus, the performance of the present algorithm in general cases improves by many orders of magnitude over that exhibited by other methods available at present.

Our approach is related to the well-known $k$-space method [1], which is based on representation of the solution by its Fourier transform, in cartesian coordinates, in all of space. In this case, truncation of the Fourier transform at wavenumbers $K$ leads to errors of the order of $1 / K$, and thus, the error in the $k$-space method is of order one in the mesh-size (compare analysis and numerical results given in [4]). Our method takes advantage of the highly accurate approximations

Manuscript received October 5, 1999; revised June 26, 2000. This work was supported by the Air Force Office of Scientific Research, Air Force Materials Command, USAF under Grant F49620-96-1-0008. The work of O. P. Bruno was supported by NSF under Contracts DMS-9523292 and the Air Force Office of Scientific Research, Air Force Materials Command, USAF under Grant F49620-99-1-0010 and F49620-99-1-0193.

O. P. Bruno is with Applied Mathematics, Caltech, Pasadena, CA 91125 USA.

A. Sei is with the Ocean Technology Department, TRW, Redondo Beach, CA 90266 USA.

Publisher Item Identifier S 0018-926X(00)10843-9. provided by Fourier series in the periodic case, and it thus achieves the claimed high-order accuracy, see Section IV.

\section{PREliminaries}

We consider a scattering configuration in which a bounded scatterer $\Omega$ is contained within a radius $R$ from the origin; the relevant nondimensional size is given by $\kappa R=2 \pi R / \lambda$. The refractive index $n(x)$ varies arbitrarily in $\Omega$ and $n(x)=1$ outside the scatterer. Thus, setting $m(x)=1-n^{2}(x)$, for $|x|>R$ we have $n(x)=1$ and $m(x)=0$. For a given incident field $u^{i n c}$, the total field $u$, equal to the sum of $u^{i n c}$ and the scattered field $u^{s}$, is a solution of Helmholtz's equation $\Delta u+\kappa^{2} n^{2}(x) u=0$ or, alternatively, a solution of the Lippmann-Schwinger integral equation

$$
u(x)=u^{i n c}(x)-\frac{i \kappa^{2}}{4} \int_{\Omega} H_{0}^{1}(\kappa|x-y|) m(y) u(y) d y
$$

where $H_{0}^{1}$ is the Hankel function of the first kind; see, e.g., [2]. We solve this equation by the iterative technique GMRES [3], which requires repeated evaluations of the right-hand side (RHS) of (1) for given inputs $u$. The accuracy of the calculation is limited by that of the integral evaluations; thus our focus on high-order singular quadrature rules.

\section{NUMERICAL METHOD}

Our algorithm is based on use of the addition theorem, which provides a mean to integrate to high order the singular integrand in (1). We thus use polar coordinates $x=a e^{i \psi}$ and $y=r e^{i \theta}$ so that the integral on the RHS of (1) becomes

$$
\begin{aligned}
K[u](a, \psi)= & -\frac{i \kappa^{2}}{4} \int_{0}^{R} r d r \int_{0}^{2 \pi} \\
& \cdot H_{0}^{1}\left(\kappa\left|a e^{i \psi}-r e^{i \theta}\right|\right) m(r, \theta) u(r, \theta) d \theta .
\end{aligned}
$$

Expanding the kernel in (2) via the addition theorem for the Hankel function

$$
\begin{aligned}
H_{0}^{1}\left(\kappa\left|a e^{i \psi}-r e^{i \theta}\right|\right) & =\sum_{\ell=-\infty}^{+\infty} \mathcal{J}_{\ell}(a, r) e^{i \ell(\psi-\theta)} \\
\mathcal{J}_{\ell}(a, r) & =J_{\ell}(\kappa \min (a, r)) H_{\ell}^{1}(\kappa \max (a, r))
\end{aligned}
$$

and introducing the Fourier series expansion $K[u](a, \psi)=$ $\sum_{\ell=-\infty}^{\infty} K_{\ell}[u](a) e^{i \ell \psi}$ we then obtain the expression

$$
\begin{aligned}
& K_{\ell}[u](a) \\
& \quad=-\frac{i \kappa^{2}}{4} \int_{0}^{R} \mathcal{J}_{\ell}(a, r) r d r \int_{0}^{2 \pi} m(r, \theta) u(r, \theta) e^{-i \ell \theta} d \theta
\end{aligned}
$$

which is a centerpiece of our algorithm. The discontinuities of $m$ are the only obstacles to high-order integration in $\theta$. To deal with such discontinuities we approximate $m$ by a truncated Fourier series $m^{F}(a, \psi)=\sum_{\ell=-F}^{F} m_{\ell}(a) e^{i \ell \psi}$. For finite $F, m^{F}$ provides a smooth approximation of $m$ and therefore allows for high-order $\theta$ integration. (As our numerical results demonstrate, extremely accurate approximations of near fields and far fields can be obtained for relatively small values of $F$; this fact is what makes our approach 

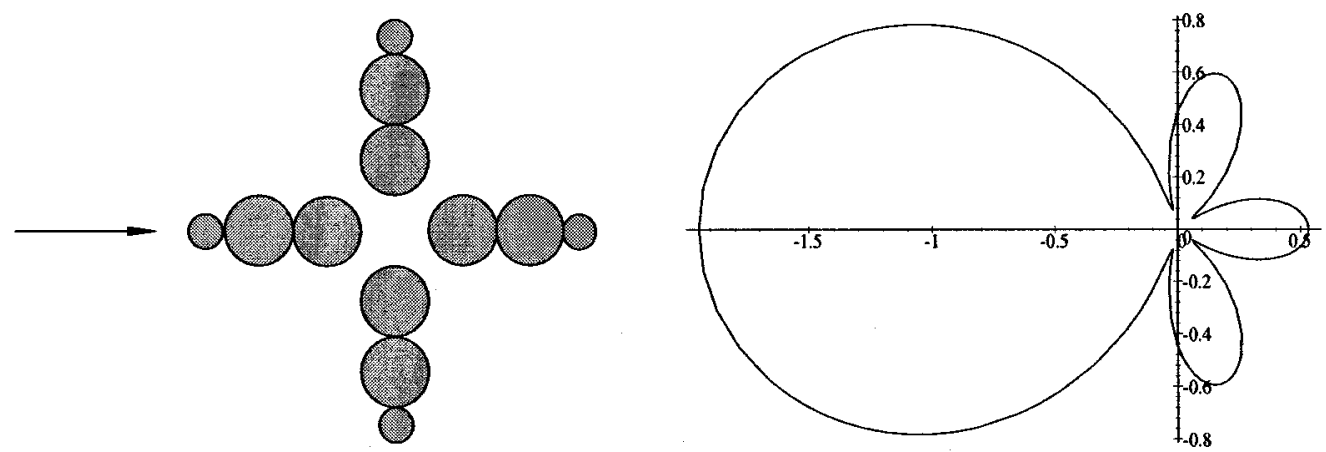

Fig. 1. A scattering configuration and its far-field pattern.

TABLE I

ERroRs AND TIMINGS FOR THE CONFIGURATION OF FIg. $1: N_{p}=3$ AND $\kappa R=3$ SHOWING A Linear DePENDENCE OF INTEGRATION TIMES AND OVERHEAD $M \cdot N_{r}$

\begin{tabular}{c|c|c|c|c|c|c|c|c|c}
\hline$n^{2}$ & $M$ & $N_{\theta}$ & $N_{r}$ & \# Iter & OH(s) & NO(ms) & Tot. Time(s) & NT(ms) & Error (Far Field) \\
\hline 2 & 10 & 121 & 23 & 2 & 0.593 & 2.5 & 1.934 & 72 & $1(-3)$ \\
\hline 2 & 20 & 131 & 31 & 2 & 1.273 & 2.0 & 5.007 & 75 & $1(-4)$ \\
\hline 2 & 30 & 141 & 43 & 3 & 2.332 & 1.8 & 12.794 & 67 & $3(-5)$ \\
\hline 72 & 10 & 121 & 19 & 2 & 0.611 & 3.2 & 8.678 & 52 & $3(-1)$ \\
\hline 72 & 20 & 141 & 33 & 3 & 1.666 & 2.5 & 46.952 & 57 & $7(-2)$ \\
\hline 72 & 30 & 225 & 63 & 6 & 4.110 & 2.2 & 264.233 & 57 & $7(-3)$ \\
\hline
\end{tabular}

viable.) Analogously, we define $u^{M}(r)=\sum_{l=-M}^{M} u_{\ell}(r) e^{i \ell \theta}$, $I_{\ell}^{F, M}(r)=\int_{0}^{2 \pi} m^{F}(r, \theta) u^{M}(r, \theta) e^{-i \ell \theta} d \theta$ and

$$
K_{\ell}^{F, M}[u](a)=-\frac{i \kappa^{2}}{4} \int_{0}^{R} \mathcal{J}_{\ell}(a, r) I_{\ell}^{F, M}(r) r d r .
$$

Now we note that the integrand defining $I_{\ell}^{F, M}(r)$ is a periodic regular function of $\theta$. Thus, the $\theta$ integration can be performed by means of the trapezoidal rule, which, in addition to being exponentially accurate, can be evaluated with a reduced operation count by means of the FFT. Our integration problem now reduces to evaluation of radial integrals via high order quadrature. To compute the integral in (5) we note that

$$
\begin{aligned}
K_{\ell}[u](a)= & H_{\ell}^{1}(\kappa a) \int_{0}^{\min (a, R)} J_{\ell}(\kappa r) I_{\ell}^{F, M}(r) r d r \\
& +J_{\ell}(\kappa a) \int_{\min (a, R)}^{R} H_{\ell}^{1}(\kappa r) I_{\ell}^{F, M}(r) r d r .
\end{aligned}
$$

We assume $I_{\ell}^{F, M}(r)$ is differentiable to a sufficiently high order. This is not a restrictive condition since appropriate changes of variables can be used to resolve the singularities that may occur in this function. The radial integration algorithm we have devised is based on polynomial interpolations. To evaluate $K_{\ell}[u]$ we divide the radial integration domain in a number $N_{i}$ of interpolation intervals. Within each interval $u_{\ell}(r)$ is interpolated by a polynomial of degree $N_{p}-1$, thus yielding errors of order $h^{N}$ for a mesh size of order $h$. For adequate values of $N_{p}$ direct integration of these polynomials provides accurate approximations of $K_{\ell}[u]$. The overall complexity of the method is $N \log (N)$, where calling $N_{r}=N_{i}\left(N_{p}-1\right)+1$ the number of points in the discretizations for the $r$ variable, $N=N_{r} M$. (Note that the angular discretization parameter $N_{\theta}$ does not appear explicitly in this complexity estimate: the number $M$ of Fourier modes is used instead.)

\section{NUMERICAL RESULTS}

We have produced solutions for a large class of scattering configurations, including smooth scatterers as well as scatterers with singularities such as corners and cusps. In the case of a circular cylinder where analytical solutions are available, double-precision accuracies were obtained in short computing times. A nontrivial comparison with an exact solution as discussed below is also presented, showing convergence consistent with that obtained in the more complex cases. In what follows we present calculations for one of the most challenging geometric configurations we have treated, containing discontinuities and cusps in the distribution of refractive index, see Fig. 1. We first show results for low values of the refractive index, $n^{2}=2$, and then, following [4], for the much larger value $n^{2}=72$. In fact there is no limit to the complexity of the problem that can be treated provided appropriate discretizations are utilized; see Table I. All the reported computations were performed on a single-processor 200-MHz Silicon Graphics R-10000 desktop computer.

We thus consider the scattering configuration depicted in Fig. 1, formed by 12 touching circles of refractive index $n, 8$ of radius 0.5 , and 4 of radius 0.25 , in such a way that the smallest circle containing the scatterer has radius $R=3$. The cusps in this geometry are well known to present severe difficulties to other algorithms. For such a dis- 
continuous refractive index it is necessary to use Fourier smoothing; the value $F=100$ was used here. (Thorough numerical tests, including comparison with the exact solution mentioned below as well as convergence tests, have shown that the values of the smoothing parameter $F$ we used give converged solutions within the error bars quoted.) Table I shows convergence studies for $n^{2}=2$ and $n^{2}=72$. Errors in the table (maximum absolute values in the far field) were obtained by comparison with a much more refined discretization: $F=200, M=100$, $N_{r}=181$, and $N_{\theta}=301$ in the case $n^{2}=2$, and $F=200, M=40$, $N_{r}=301$, and $N_{\theta}=295$ for $n^{2}=72$. Near field errors are generally larger by a factor of 10 . As claimed, our algorithm resolves the $n^{2}=2$ configuration with an error of order $10^{(-5)}$ in a 13-s run. The restart parameter required by GMRES was taken to equal 4 in the case $n^{2}=2$ and to equal 40 for $n^{2}=72$. OH denotes the overhead, and $\mathrm{NO}$ and NT denote the normalized quantities $\mathrm{NO}=10^{3} \mathrm{OH} /\left(M \cdot N_{r}\right)$ $\mathrm{NT}=10^{3}($ Time $-\mathrm{OH}) /\left(\right.$ Restart $\cdot$ Iter $\left.\cdot M \cdot N_{r}\right)$. In order to insure that these tests give an accurate measure of the error we applied the same procedure to an off-center circle for which an analytical solution is known. This geometry provides a nontrivial test for our solver since it involves discontinuities in the refractive index within the domain of integration. Our test circle has radius one and is centered at $(2,0)$. For $n^{2}=2$ using $M=10,20$, and 40 our solver yields errors of $2(-4)$, $3(-5)$, and $4(-6)$, respectively. For $n^{2}=72$ using $M=10,20$, and 40 modes we obtained errors of $3(0), 2(-2)$, and $2(-3)$, respectively, in good agreement with the orders of the errors shown in Table I.

\section{REFERENCES}

[1] N. N. Bojarski, "The $k$-space formulation of the scattering problem in the time domain," J. Opt. Soc. Amer., vol. 72, pp. 570-584, 1982.

[2] D. Colton and R. Kress, Inverse Acoustic and Electromagnetic Scattering Theory. New York: Springer-Verlag, 1992.

[3] Y. Saad and M. H. Schultz, "GMRES: A generalized minimal residual algorithm for solving non-symmetric linear systems," SIAM J. Sci. Statist. Comput., vol. 7, pp. 856-869, 1986.

[4] P. Zwamborn and P. Van den Berg, "Three dimensional weak form of the conjugate gradient FFT method for solving scattering problems," IEEE Trans. Microwave Theory Tech., vol. 40, pp. 1757-1766, Sept. 1992.

\section{A Low-Profile Conical Beam Loop Antenna with an Electromagnetically Coupled Feed System}

\author{
H. Nakano, K. Fujimori, and J. Yamauchi
}

\begin{abstract}
The radiation characteristics of a low-profile loop antenna are evaluated using the method of moments (MoM). The loop having a circumference of approximately two wavelengths is electromagnetically coupled to a bent feed line and radiates a circularly polarized conical beam. The frequency bandwidth for a 3-dB axial ratio criterion is calculated to be approximately $0.5 \%$ for an antenna height of $h=0.064$ wavelengths. Over the same bandwidth, the input impedance is approximately $50 \mathrm{ohms}$ and the gain is approximately $7 \mathrm{~dB}$.
\end{abstract}

Index Terms-Electromagnetically coupled feed, loop.

\section{INTRODUCTION}

It is well known that a loop antenna is a linearly polarized (LP) radiation element [1], [2]. Recent theoretical study has revealed that, when two perturbation elements are added to a one-wavelength circumference loop [3], an LP axial beam can be changed to a circularly polarized $(\mathrm{CP})$ axial beam.

Automobile communication systems often require a $\mathrm{CP}$ conical beam antenna in addition to a $\mathrm{CP}$ axial beam antenna. For this requirement, this letter presents a low-profile loop antenna that radiates a $\mathrm{CP}$ conical beam. For CP conical beam formation, a single perturbation element is added to a loop whose circumference is approximately two wavelengths.

Attention is paid to input impedance matching. To reduce the highinput impedance of the conventional loop antenna fed directly from a coaxial line, an electromagnetically coupled feed system is proposed. The feed line in this system is not in contact with the loop, leading to straightforward impedance matching.

\section{DISCUSSIONS}

Fig. 1 shows the configuration and coordinate system of a low-profile loop antenna. The loop is made of a thin wire of radius $\rho$. The loop of circumference $C$, backed by a conducting ground plane, has a perturbation element of length $\Delta L$ at point $b$. The distance from the ground plane to the loop section (antenna height) and the angle made by the $x$ axis with line $o^{\prime}-b$ (perturbation angle) are designated as $h$ and $\phi_{b}$, respectively. The loop is supported by a dielectric material of relative permittivity $\varepsilon_{r}$.

The feed system consists of a bent wire $f-c-d$ of radius $\rho_{F}$, which is electromagnetically coupled to the loop (EM-coupled feed). The curved section of the feed wire $c-d$ is parallel to the loop and located just under the loop. The vertical section $f-c$ and the parallel section $c-d$ have lengths of $L_{V}$ and $L_{H}$, respectively.

Throughout this letter, the following parameters are chosen to be as follows: $\rho=0.003 \lambda_{0}=0.6 \mathrm{~mm}, h=0.064 \lambda_{0}=12.8 \mathrm{~mm}, \varepsilon_{r} \approx 1$ (honeycomb material is used for a spacer), and $\rho_{F}=0.0031 \lambda_{0}=0.62$ $\mathrm{mm}$, where $\lambda_{0}(=200 \mathrm{~mm})$ is the wavelength at a design frequency of $f_{0}=1.5 \mathrm{GHz}$.

The loop circumference, perturbation element length, and bent wire length are initially chosen to be $C=2 \lambda_{0}, \Delta L=0.025 \mathrm{C}$, and $L_{V}+$ $L_{H}=\lambda_{0} / 4$, respectively. Then these values are optimized such that 